\title{
Polyphenolic extract association with renin inhibitors in experimental arterial hypertension
}

\author{
Manuela Ciocoiu $^{1}$, Magda Badescu ${ }^{1 *}$, Oana Badulescu ${ }^{1}$, Dana Tutunaru ${ }^{2}$, Laurentiu Badescu ${ }^{3}$ \\ ${ }^{1}$ Department of Pathophysiology, Faculty of Medicine, University of Medicine and Pharmacy “Gr. T. Popa”, Iasi, Romania \\ ${ }^{2}$ Department of Biochemistry, University "Dunarea de Jos”, Galati, Romania \\ ${ }^{3}$ Department of Cellular and Molecular Biology, University of Medicine and Pharmacy “Gr. T. Popa”, Iasi, Romania \\ Email: ㅆagda.badescu@gmail.com
}

Received 2 March 2013; revised 4 April 2013; accepted 18 April 2013

Copyright (C) 2013 Manuela Ciocoiu et al. This is an open access article distributed under the Creative Commons Attribution License, which permits unrestricted use, distribution, and reproduction in any medium, provided the original work is properly cited.

\begin{abstract}
Various epidemiological studies have shown an inverse association between the consumption of polyphenols or polyphenol-rich foods and the risk of cardiovascular diseases. The purpose of the study was to emphasize the effects of the association between the renin inhibitor and the polyphenolic extract on biochemical parameters and systolic and diastolic blood pressure. The polyphenols are extracted from isolated and purified vegetable material represented by the mature fruit of the Aronia melanocarpa. The experiment was performed on the arterial hypertension model. In the arterial hypertensive model the cardioprotective effects of the polyphenolic extract from Aronia melanocarpa are represented by the antioxidant, hypocolesterolemiant intervention. Polyphenolic extract from Aronia melanocarpa reduce systolic and diastolic arterial tension values in rats with druginduced hypertension, a phenomenon more pronounced when polyphenols are associated with Aliskiren (renin inhibitor). The combination between the Aliskiren and polyphenolic extract produce superior hypolipidemic and antioxidant effects than in the case of separate administration within experimental arterial hypertension induced in the murine model.
\end{abstract}

Keywords: Arterial Hypertension; Aliskiren; Aronia melanocarpa; Oxidative Stress

\section{INTRODUCTION}

Polyphenols and other food phenolics are the subject of increasing scientific interest because of their possible beneficial effects on human health. The fruit of the Aronia melanocarpa, a shrub of the rosaceous family, has a

\footnotetext{
"Corresponding author.
}

dark purple peel and contains high levels of flavonoids and phenolic acids. Main flavonoid subgroups in chokeberry are proanthocyanins, anthocyanins, flavornols and catechins [1].

More evidence for a protective role of polyphenols against cardiovascular diseases arose from a number of clinical trials [2], experiments on animal models and mechanistic studies [3]. Various epidemiological studies have shown an inverse association between the consumption of polyphenols or polyphenol-rich foods and the risk of cardiovascular diseases [4]. Since polyphenols are redox active compounds they may also cause increased radical formation if they uncouple electron pathways in the body or if they chelate transition metals in such a way that they become more reactive like in the experimental Fenton oxidation systems [5].

The purpose of the study was to emphasize the effects of the association between the renin inhibitor and the polyphenolic extract on biochemical parameters and systolic and diastolic blood pressure.

\section{MATERIAL AND METHODS}

Ripe berries of Aronia melanocarpa Michx. (Rosaceae, chokeberry) were shade-dried at room temperature for one week. Dried chokeberries (100 g) were chopped into small pieces and extracted with $3 \times 700 \mathrm{ml}$ ethanol using a magnetic stirrer (FALC F30ST), each time for $3 \mathrm{~h}$. The combined extracts were taken to dryness by evaporation under reduced pressure (BÜCHI R-210 rotavapor, BÜCHI V-850 vacuum controller, BÜCHI V-700 vacuum pump). Total phenolics quantification was performed by FolinCiocalteu method. The absorbance was measured at 765 $\mathrm{nm}$ after $2 \mathrm{~h}$ of incubation at room temperature. A calibration curve was plotted using gallic acid as standard. The total phenolic content was expressed as mg gallic acid equivalents/g extract. Sample was assayed in tripli- 
cate and the results were given as the mean \pm standard deviation.

The research was performed on Wistar white rats, with an average weight of $250-280 \mathrm{~g}$, which were divided into 6 groups of 12, namely: Group W-control, normal animals, that didn't receive natural polyphenols; Group AHT-animals which were administered L-NAME 40 $\mathrm{mg} / \mathrm{kg}$ body/day, i.p., at every 2 days, for 8 weeks; Group PA - animals that were administered polyphenols under the form of solution, from the extract obtained from the Aronia melanocarpa fruit, with a dosage of $0.045 \mathrm{~g} / \mathrm{Kg}$ body, p.o. (by tube feeding), at every 2 days, for 8 weeks; Group AHT + PA animals which were administered polyphenols in the dosage mentioned p.o. at every 2 days, concomitantly with L-NAME, for 8 weeks; Group AHT + Alisk animals with AHT induced by L-NAME, that were given Aliskiren $30 \mathrm{mg} / \mathrm{kg}$ body/day, administered s.c. for 8 weeks; Group AHT + Alisk + PA animals with AHT that were given Aliskiren and polyphenols in the above mentioned dosage, for 8 weeks.

The in vivo experiments, on experimental animal sample were carried out in specially equipped premises, where conditions of habitat in accordance with standard norms were created. Between experiments, the rats were kept in individual cages and were given food and water. A 12 hours light/12 hour darkness cycles was employed.

The experiments on murin model complied with the ethical norms in experimental research, yet also with the terms enforced by the Iasi "Grigore T. Popa" University of Medicine and Pharmacy Commission of Bioethics. At the same time, the EU norms concerning lab rats were taken into consideration (the Directive of the European Council Committee 86/609/EEC).

Ceruloplasmin determination was performed on an EOS BRAVO automatic analyzer. Uric acid determination was carried out by means of Siedel's modified colorimetric method.

The exploration of the lipid profile included the measurement by photocolorimetry, in the serum obtained after separation, of the concentration of total cholesterol (Ch-T), of total lipids (LT), [the method with sulfovaniline], of high-density lipoproteins (HDL) (Lopes-Virella, 1977), of low-density lipoproteins (LDL) [according to the Friedewald formula] for all the animals included in the experiment.

The rat blood pressure values were recorded using a CODA $^{\mathrm{TM}}$ system purchased from Kent Scientific Corporation, which uses a non-invasive blood pressure measuring method. It records the blood volume-pressure by a band attached to the tail, homologated by Bland-Altman testing designed to reveal conformity with an invasive method (radiotelemetry), which enjoys proven accuracy, yet it is difficult to use in our study. The method is also recommended by the AHA in its blood pressure measur- ing guide for laboratory animals. The actual experiment consists of performing at least 6 blood pressure measurements in each laboratory animal, data collection by means of the $\mathrm{CODA}^{\mathrm{TM}}$ software and subsequent data processing.

Statistical Data Interpretation. All the data are shown as mean value \pm standard error of the mean (SEM). In order to assess the normal distribution of the groups, Shapiro-Wilk test was performed. Additionally, Levene test was performed to confirm the homoscedasticity of the groups, followed by ANOVA and paired or unpaired t-test to reveal the pairs of groups that differ biostatistical significantly in term of means. Statistical data interpretation considered the corresponding differences for a given significance threshold: $\mathrm{p}>0.05$ statistically insignificant; $\mathrm{p}<0.05$ statistically significant; $\mathrm{p}<0.01$ strong statistical significance; $\mathrm{p}<0.001$ very strong statistical significance.

\section{RESULTS AND DISCUSSION}

The lowest average values of the ceruloplasmin have been recorded in the HTA group. The highest average values of the ceruloplasmin have been recorded in the witness group and in the groups treated with Aronia melanocarpa.

Note that all the groups present higher average values of the ceruloplasmin compared to the hypertensive group, which suggests the antiradical beneficial effect of the ceruloplasmin. The most efficient administration proved to be Aronia melanocarpa, which determines average values of the ceruloplasmin close to those recorded in witness group (Figure 1).

The ceruloplasmin and uric acid were determined as nonspecific oxidative stress markers. Statistically speaking, the uric acid values remain significantly high both in the AHT group, and in the AHT + PA group in comparison with control group (Figure 2).

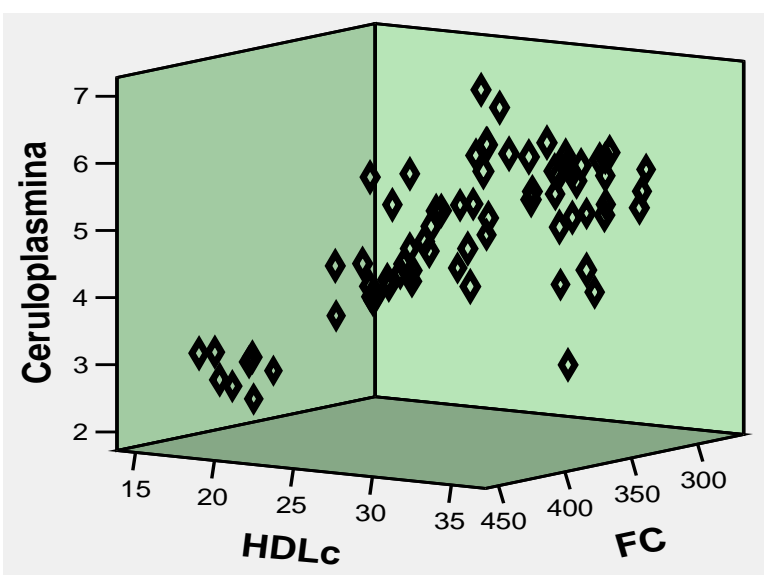

Figure 1. Correlation of the ceruloplasmin individual values with the cardiac frequency (FC) and HDL-col. 
The ability of dietary polyphenols to generate both NO and ROS in endothelial cells and activate ARE/EpRE (Antioxidant response element/Electrophile response element) mediated gene expression underlies their cardioprotective properties [6].

Dietary polyphenols may counteract oxidative stress in vascular and inflammatory diseases [7] by modulating key redox sensitive gene transcription via NF- $\kappa \mathrm{B}$ and Nrf2/ARE [8] signaling pathways. The balance between antioxidant and pro-oxidant characteristics of polyphenols have been attributed not only to their structural features, but also to the concentration, suggesting induction of antioxidant defence metabolism by low concentrations and ROS production at high concentrations [9].

It should also be noted that the total cholesterol and triglycerides-lowering activity of Aronia melanocarpa extracts was found in the case of rats fed with standard, non-hypercholesterolemic diet supplemented with high doses of chokeberry anthocyanins for 4 weeks [10].

When comparing total cholesterol and LDL-col levels, the results show that these are significantly higher in the AHT group than in the $\mathrm{W}$ group. There are significant improvements taking place against the dislipidemia occurring in arterial hypertention as a result of the administration of polyphenols extracted from Aronia melanocarpa fruit. The serum LDL-col levels in the AHT + PA group were kept within normal limits by the polyphenolic protection.

The highest average values of the total cholesterol have been recorded in the AHT group, significantly higher than in the other investigated groups. The lowest average values of the total cholesterol have been recorded in the $\mathrm{AHT}+$ Alisk + PA group, significantly lower than those recorded in AHT and W groups. From the viewpoint of the variability coefficient (\%), the mean values obtained are typical of the series considered (Figure 3).

Research comparing the AHT + PA, AHT + Alisk + PA and AHT groups shows that the HDL-col is significantly higher in the first and second group (Figure 4). The in-

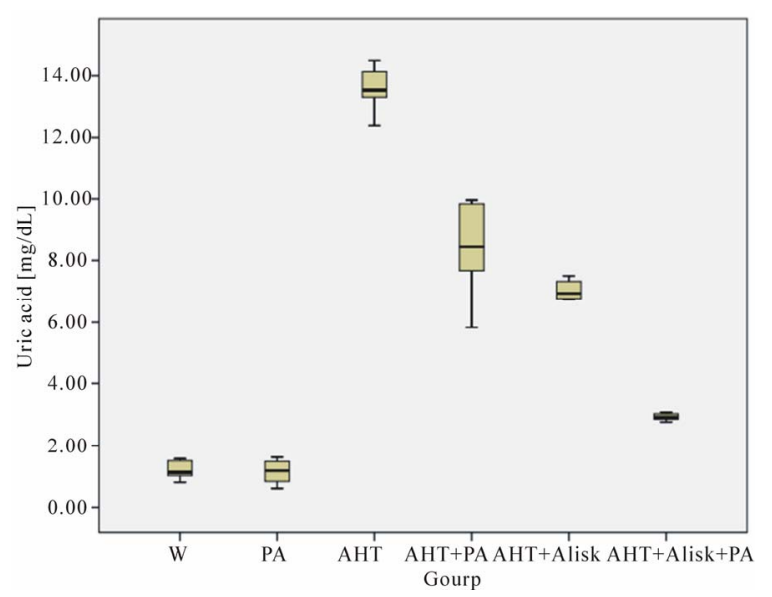

Figure 2. The box-and-whisker plot of uric acid. dividual values of the total cholesterol have presented direct correlations with the systolic blood pressure, moderated as intensity, for the PA $(r=+0.58)$ and AHT + PA $(r=+0.76)$ groups.

In a combined therapy, chokeberry extracts were given as supplements with the diet of patients after myocardial infarction, as an addition to the statin treatment. Compared to the control group, treated only with statins, patients receiving additional Aronia extract for 6 weeks had significantly lower LDL-col oxidation status as well as reduced levels of serum 8-isoprostanes and increased adiponectin levels, which indicate diminished oxidative stress and reduced endothelial inflammation [11].

The systolic and diastolic blood pressures, as well as their calculated mean, were measured. The Shapiro-Wilk test was positive, which supports sample normality, and the descriptive statistics and box-and-whisker plots are shown in Figures 5-7.

The Levene Test confirmed group homoscedasticity, whereas the ANOVA test revealed a significant difference between the means of the 4 groups, as concerns sys-

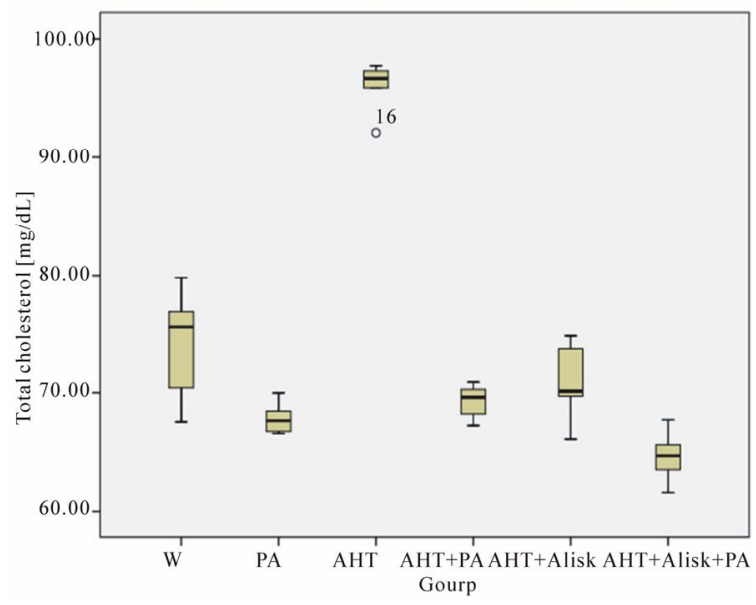

Figure 3. The box-and-whisker plot of total cholesterol.

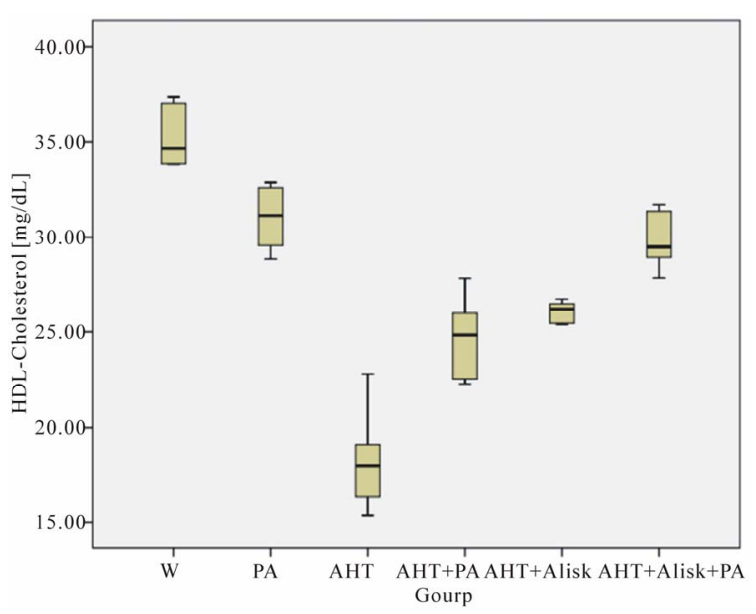

Figure 4. The box-and-whisker plot of HDL-cholesterol. 


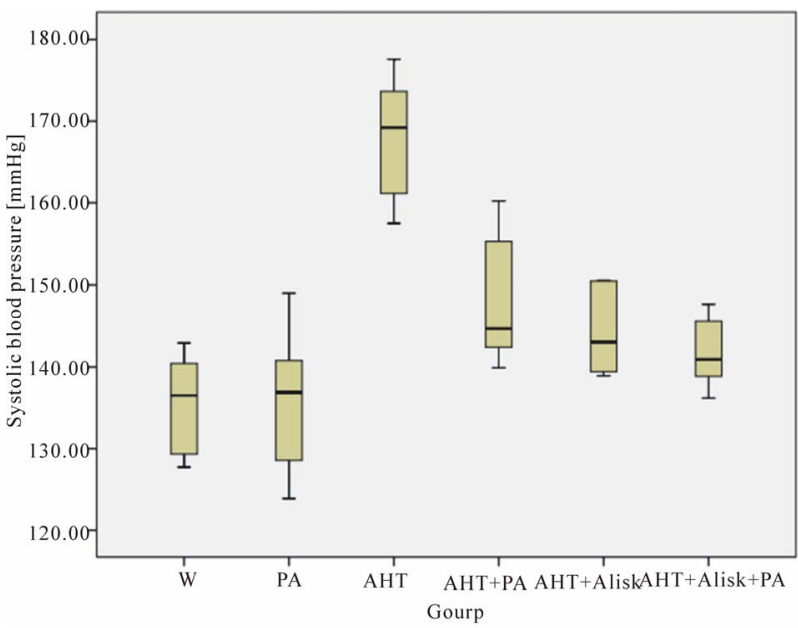

Figure 5. The box-and-whisker plot of systolic blood pressure.

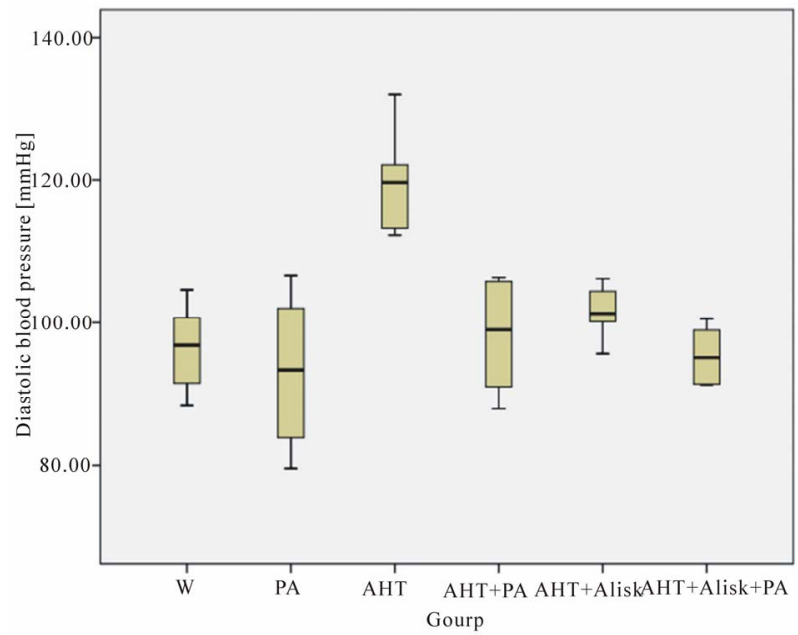

Figure 6. The box-and-whisker plot of diastolic blood pressure.

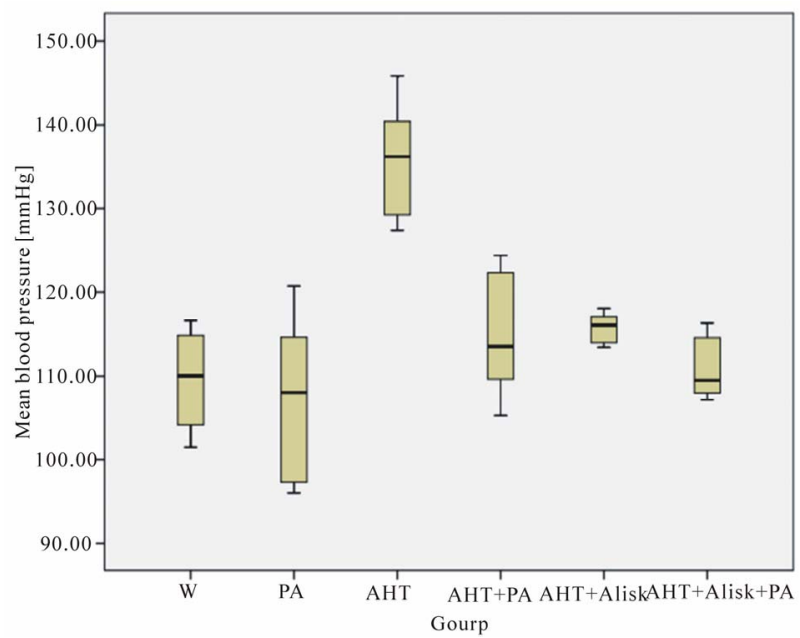

Figure 7. The box-and-whisker plot of mean blood pressure.

tolic and diastolic blood pressure. All the measured blood pressure components revealed a bio-statistically signifi- cant $(\mathrm{p}<0.05)$ blood pressure drop between the AHT and the AHT $+\mathrm{P}$ groups.

Polyphenolic extract from Aronia melanocarpa reduce systolic and diastolic arterial tension values in rats with drug-induced hypertension, a phenomenon more pronounced when polyphenols are associated with Aliskiren (renin inhibitor).

Our understanding of endogenous mechanisms of hypertension by oxidative processes has advanced greatly in the last decade, yet the description of the molecular action of predisposing factors must be further elucidated to prevent and properly treat cardiovascular diseases.

\section{CONCLUSION}

In the arterial hypertensive model the cardio-protective effects of the polyphenolic extract from Aronia melanocarpa are represented by the antioxidant, hypocolesterolemiant intervention. The results prove that oxidative stress is considerably lower, statistically speaking, in rats with hypertension provided with Aliskiren and natural polyphenolic protection from Aronia melanocarpa fruits, than in the rats belonging to the control group. The combination between the Aliskiren and polyphenolic extract produce superior hypolipidemic and antioxidant effects than in the case of separate administration within experimental arterial hypertension induced in the murine model.

\section{ACKNOWLEDGEMENTS}

The work was supported by the Romanian Ministry of Education and Research, CNCSIS, plan PN2, program IDEI, section PCE, research grant ID-2519/2008.

\section{REFERENCES}

[1] Oszmianski, J. and Wojdylo, A. (2005) Aronia melanocarpa phenolics and their antioxidant activity. European Food Research and Technology, 1, 1-5.

[2] Pascual-Teresa, S. and Sanchez-Ballesta, M.T. (2008) Anthocyanins: From plant to health. Phytochemistry Reviews: Proceedings of the Phytochemical Society of Europe, 7, 281-299.

[3] Mennen, L.I., Sapinho, D. and De Bree, A. (2004) Consumption of foods rich in flavonoids is related to a decreased cardiovascular risk in apparently healthy French women. The Journal of Nutrition, 134, 923-926.

[4] Bell, D.R. and Gochenaur, K. (2006) Direct vasoactive and vasoprotective properties of anthocyanin-Rich extracts. Journal of Applied Physiology, 100, 1164-1170. doi:10.1152/japplphysiol.00626.2005

[5] Yao, L.H., Jiang, Y.M., Shi, J., Tomas-Barberan, F.A., Datta, N. and Singanusong, R. (2004) Flavonoids in food and their health benefits. Plant Foods for Human Nutrition, 59, 113-122. 
[6] Lee-Hilz, Y.Y., Boerboom, A.M., Westphal, A.H., Berkel, W.J., Aarts, J.M. and Rietjens I.M. (2006) Pro-oxidant activity of flavonoids induces EpRE mediated gene expression. Chemical Research in Toxicology, 19, 1499-1505. doi:10.1021/tx060157q

[7] Rahman, I., Biswas, S.K. and Kirkham, P.A. (2006) Regulation of inflammation and redox signaling by dietary polyphenols. Biochemical Pharmacology, 72, 1439-1452. doi:10.1016/j.bcp.2006.07.004

[8] Hernandez-Montes, E., Pollard, S.E., Vauzour, D., JofreMontseny, L., Rota, C. and Rimbach, G. (2006) Activation of glutathione peroxidase via Nrf1 mediates genistein's protection against oxidative endothelial cell injury. Biochemical and Biophysical Research Communications, 346, 851-859. doi:10.1016/j.bbrc.2006.05.197

[9] Masella, R., Di Benedetto, R., Vari, R., Filesi, C. and
Giovannini, C. (2005) Novel mechanisms of natural antioxidant compounds in biological systems: Involvement of glutathione and glutathione-related enzymes. The Journal of Nutritional Biochemistry, 16, 577-586. doi:10.1016/j.jnutbio.2005.05.013

[10] Kokotkiewicz, A., Jaremicz, Z. and Luczkiewicz, M. (2010) Aronia plants: A review of traditional use, biological activities, and perspectives for modern medicine. Journal of Medicinal Food, 13, 255-269. doi:10.1089/jmf.2009.0062

[11] Naruszewicz, M., Laniewska, I., Millo, B. and Dluzniewski, M. (2007) Combination therapy of statin with flavonoids rich extract from chokeberry fruits enhanced reduction in cardiovascular risk markers in patients after myocardial infarction. Atherosclerosis, 194, e179-e184. doi:10.1016/j.atherosclerosis.2006.12.032 